\title{
Fabrication of the Crystalline ITO Pattern by Picosecond Laser with a Diffractive Optical Element
}

\author{
C.W. Chien and C.W. Cheng* \\ ITRI South Campus, Industrial Technology Research Institute, \\ No. 8, Gongyan Rd., Liujia District, Tainan 734, Taiwan \\ E-mail: CWCheng@itri.org.tw (C.W. Cheng)
}

\begin{abstract}
This study presents the fabrication of crystalline indium tin oxide (c-ITO) patterns on glass substrate using laser-induced crystallization followed by chemical etching. In the proposed approach, an amorphous ITO (a-ITO) thin film was transformed into a c-ITO structure over a predetermined area using infrared picosecond fiber laser irradiation with a $500 \mathrm{kHz}$ repetition rate, under a single laser beam or multiple laser beams by diffractive optical elements (DOE). The residual a-ITO thin film was removed by an etchant solution, in order to form a uniform c-ITO pattern. The formation was due to the heat accumulation effect provided by the high-repetition rate of picosecond laser pulses.

DOI:10.2961/jlmn.2013.02.0009
\end{abstract}

Keywords: picosecond laser, ITO, crystalline, DOE

\section{Introduction}

Indium tin oxide (ITO) is widely used as a transparent conducting material with high transparency in the visible region of the spectrum. The resistivity of an ITO pattern should be maintained as low as possible in order to obtain a high response for functional devices, such as touch panels and organic light-emitting diodes. A crystalline structure usually has a lower resistivity than an amorphous structure does; innovation in the patterning of crystalline-ITO (cITO) technology has a significant impact on both fabrication cost and processing time.

The conventional fabrication of a c-ITO pattern is based on a photolithographic process, where an amorphous-ITO (a-ITO) thin film undergoes thermal annealing at a temperature higher than $200^{\circ} \mathrm{C}$ [1]. This is a complex process that involves photoresist coating and baking, photoresist exposure and developing, etching and photoresist stripping. Thus, during the heat treatment, other layers are easily damaged. Laser ablation is a natural technology intended for use in patterning ITO film, and many researchers have investigated the ablation process with nanosecond, picosecond and femtosecond lasers [2-8]. However, the ablation process requires sufficient laser fluence to evaporate the materials. Elevated ridges of edges and ITO residue on an ablated bottom easily occur, which may cause shorting of the structure or the adjacent electrodes. To overcome the above disadvantages, the researchers utilized a nanosecond excimer laser to pattern a-ITO thin films using a crystallization effect [9]. However, the results showed that they had not succeeded in crystallizing a-ITO layers on glass substrate without cracking, due to thermal shocks. In our previous studies [10-12], a novel technique to fabricate c-ITO micro patterns from a-ITO thin film using femtosecond laser-induced crystallization, followed by chemical etching, was presented. Ridge-free and crack-less mi- cro/nano c-ITO structures were fabricated by single laser beam scanning.

In this study, we extend the experiments to the picosecond region and the fabrication of the c-ITO micro patterns from a-ITO thin film using picosecond laser-induced crystallization, followed by chemical etching is demonstrated. Crystallization under different conditions: a single laser beam with different laser powers and scanning speeds, and multiple laser beams from diffractive optical elements (DOE) are studied. The irradiated ITO film is then etched in an oxalic acid solution to remove residual aITO regions since a-ITO has a higher etching rate than cITO does [13].

The results show that, given an appropriate choice of laser parameters, crack-free c-ITO patterns can be accomplished. Furthermore, parallel crystallization of the ITO film created by applying DOE has a higher efficiency compared to single-point scanning.

\section{Experimental Setup}

Figure 1(a) presents the schematic illustration of the experimental setup. The experiments were performed using an infrared picosecond fiber laser (HE1060-5, Fianium Inc.), with a pulse width of 45 ps, a wavelength of 1064 $\mathrm{nm}$, a repetition rate of $500 \mathrm{kHz}$ and an average laser power of $5 \mathrm{~W}$. The transmitted laser beam was illuminated on a DOE of diffractive area with a diameter of $60 \mathrm{~mm}$ (DFT3L, Stocker Yale, Inc.), such that the single laser beam is divided into five beams with different angles of propagation. The angle separation between two adjacent beams is $1^{\circ}$. Figure 1(b) shows the beam profile before and after the DOE. The five beam profiles were measured together at position $50 \mathrm{~mm}$ after the DOE using a CCD-based beam profiler (Ophir Spiricon SP-620U). The average power for the incident laser beam is $0.095 \mathrm{~W}$. The average power for 
the five beams was measured separately at a distance 1500 $\mathrm{mm}$ after DOE; they are: $0.015,0.015,0.014,0.015,0.015$ $\mathrm{W}$, respectively. The efficiency is $78.9 \%$ and the power vibration is about $6.7 \%$. The laser beams passed through a galvanometric scanner (intelliSCNA 14, Scanlab $\mathrm{GmbH}$ ) with an $F$-theta telecentric lens (focal length $100 \mathrm{~mm}$ ). In the current experimental setup, due to the limited size of the scanning mirror, only the center's three laser beams after DOE can be scanned. The distance between the DOE and the scanning mirror inside the galvanometric scanner is about $230 \mathrm{~mm}$. It means that the distance between two adjacent beams irradiated at the scanning mirror is about 4 $\mathrm{mm}$. The total length of the five beams is about $16 \mathrm{~mm}$, which is larger than the scanning mirror $(12 \mathrm{~mm} \times 14 \mathrm{~mm})$. The telecentricity of the focus lens was used to convert the angle of each beam incident in the normal direction on the surface of the a-ITO coated specimen mounted on an X-Y axis stage.

A-ITO thin films, with thicknesses of $\sim 100 \mathrm{~nm}$, were deposited on glass substrates by a DC magnetron sputtering system. After the laser-induced crystallization process, the samples were immersed in a $0.05 \mathrm{~mol} / \mathrm{L}$ oxalic acid etchant, at $50^{\circ} \mathrm{C}$ for $30 \mathrm{~s}$. After etching, the processed area of the surface was observed by optical microscopy and scanning electron microscopy (SEM). The crystalline, optical properties and electrical resistively were measured by $\mathrm{X}$ ray diffraction method (XRD), spectrophotometer and four point probe station, respectively.

(a)

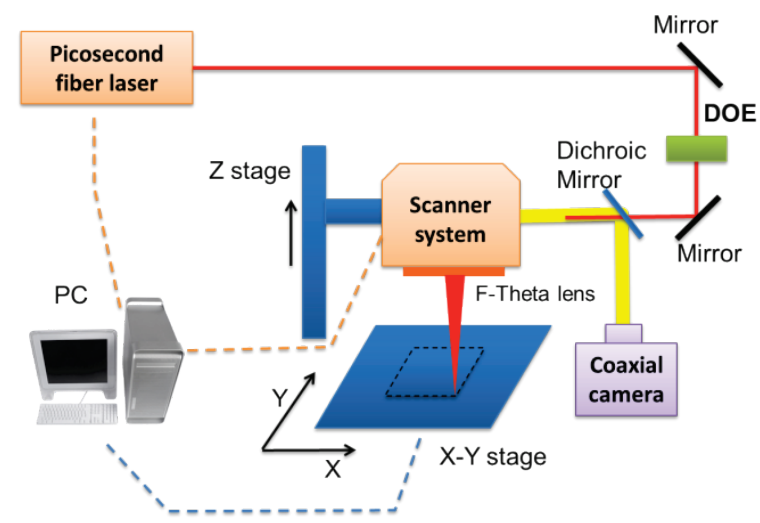

(b)

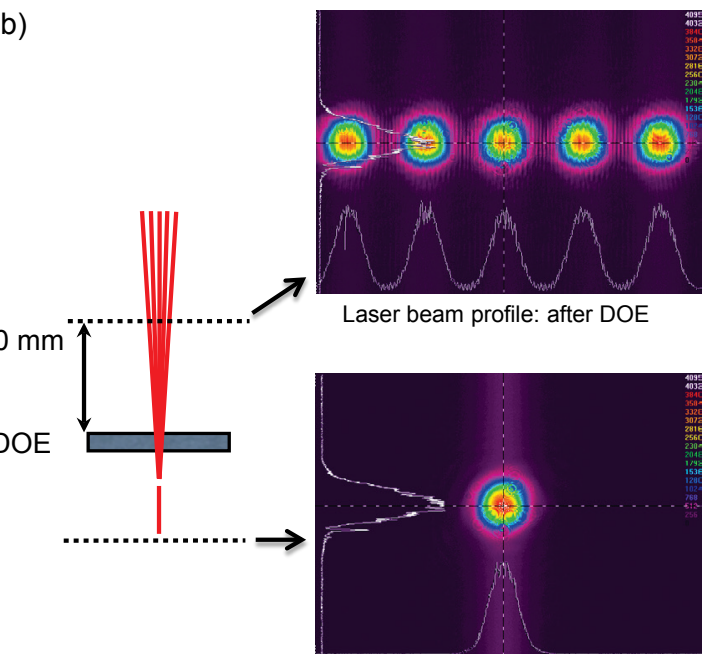

Laser beam profile: before DOE
Figure 1: (a) schematic illustration of the experimental setup and (b) the measured beam profiles before and after DOE

\section{Results and Discussion}

Figure 2(a)-(c) present microscope images showing the line patterns fabricated on a-ITO thin film surface using a single laser beam irradiation with a laser power of $0.96 \mathrm{~W}$, a pulse repetition rate of $500 \mathrm{kHz}$ and scanning speeds of $2.5 \mathrm{~mm} / \mathrm{s}, 25 \mathrm{~mm} / \mathrm{s}$ and $100 \mathrm{~mm} / \mathrm{s}$, respectively. Note that the specimen is in an unetched condition. The film within the laser irradiated area is clearly different from that within the unirradiated region. The irradiated sample was then etched, as shown in Figs. 2(d)-(f), leaving clear line structures on the glass substrate. Due to the Gaussian property of the irradiated beam profile, the middle of the scanning area is more irradiated by higher laser energy than are the edges. In Figs. 2(d)-(f), the irradiated laser energy is higher than the ablation threshold, so the center of the line is ablated. However, the outer edges are subject to lower irradiation intensity; thus, the ablated channels are bordered on either side by a c-ITO pattern.

Figure 3 presents microscope images showing the line patterns fabricated on a-ITO thin film surface using a single laser beam irradiation with a lower laser power of 0.33 $\mathrm{W}$, a pulse repetition rate of $500 \mathrm{kHz}$ and scanning speeds of $2.5 \mathrm{~mm} / \mathrm{s}, 25 \mathrm{~mm} / \mathrm{s}$ and $100 \mathrm{~mm} / \mathrm{s}$, respectively. Figs. 3(d)-(f) show that clear line patterns were patterned on the glass substrate. Since the peak energy intensity of the laser beam is higher than the crystallization threshold of a-ITO and is lower than the ablation threshold, the center of the line is crystallized.

(a)

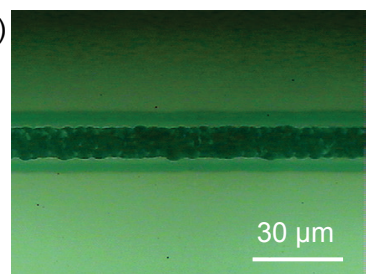

(d)

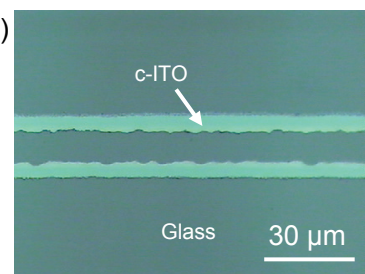

(b)

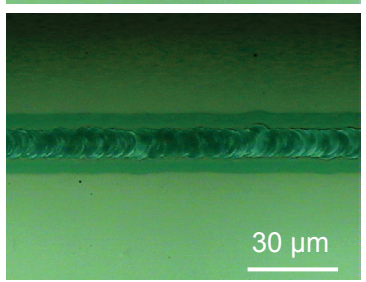

(e)

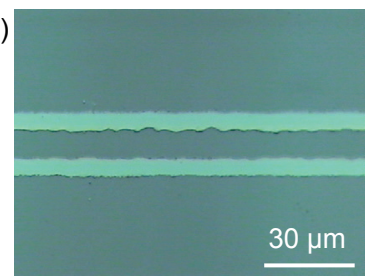

(c)

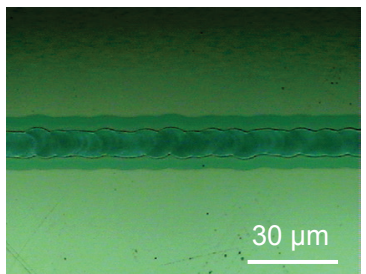

(f)

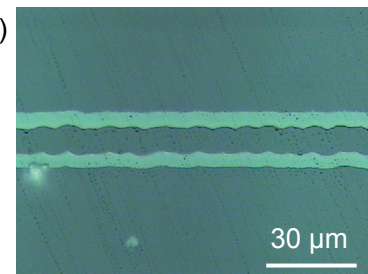

Figure 2: Microscope images of laser irradiated ITO thin film surface (a)-(c) before and (d)-(f) after etching, respectively. The line patterns were fabricated at scanning speeds of: (a)(d) $2.5 \mathrm{~mm} / \mathrm{s}$, (b)(e) $25 \mathrm{~mm} / \mathrm{s}$, and (c)(f) $100 \mathrm{~mm} / \mathrm{s}$. Note that the repetition rate was $500 \mathrm{kHz}$ and the laser power was $0.96 \mathrm{~W}$ in all cases. 
(a)

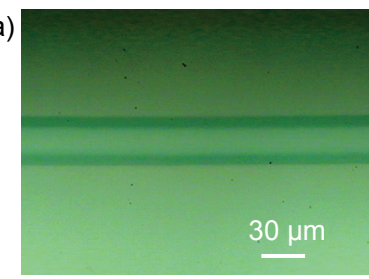

(b)

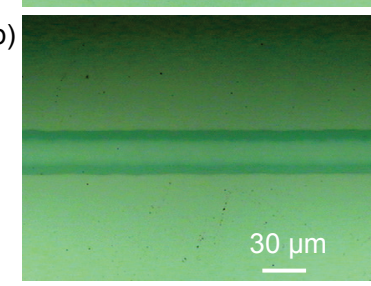

(c)

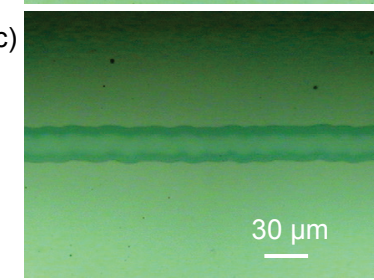

(d)

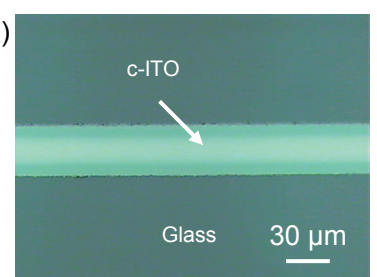

(e)

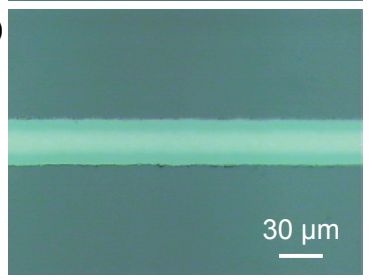

(f)

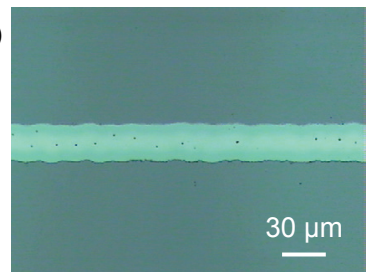

Figure 3: Microscope images of laser irradiated ITO thin film surface (a)-(c) before and (d)-(f) after etching, respectively. The line patterns were fabricated at scanning speeds of: (a)(d) $2.5 \mathrm{~mm} / \mathrm{s}$, (b)(e) $25 \mathrm{~mm} / \mathrm{s}$, and (c)(f) $100 \mathrm{~mm} / \mathrm{s}$. Note that the repetition rate was $500 \mathrm{kHz}$ and laser power was $0.33 \mathrm{~W}$ in all cases.

Figure 4 presents the SEM images of Figs. 3(d)-(f) and Figs. 2(d)-(f), respectively. Figs. 4(a)-(c) show that the width of a c-ITO line pattern decreased with increasing scanning speed, i.e., about $30 \mu \mathrm{m}, 24 \mu \mathrm{m}$ and $21 \mu \mathrm{m}$, respectively. The c-ITO line patterns were free of microcracks and showed characteristics of a smooth, unblemished surface. Thus, it appears that the high repetition rate picosecond laser (i.e., $500 \mathrm{kHz}$ ) results in a more uniform crystallization effect than that achieved using the low repetition rate laser [9]. It has been reported that the processing of certain bulk transparent glasses using a high repetition rate laser (i.e. $>200 \mathrm{kHz}$ ) prompts a heat accumulation effect, which minimizes the thermal cycling phenomenon between successive laser pulses [14]. The thermal cycling effect is therefore avoided because the local temperature remains at a high, fairly constant value throughout the annealing process rather than increasing / decreasing cyclically.

The results presented in Figs. 4(a)-(c), suggest that such a heat accumulation effect was also present in the aITO thin film specimens which were processed under the current high repetition rate $(500 \mathrm{kHz})$ picosecond laser. An effective cooling time, $t_{c}$ of the a-ITO thin film was calculated at about $521 \mu \mathrm{s}$; thus, according to [15]:

$$
t_{c}=d_{v}^{2} / D
$$

where $d_{v}$ is the diameter of the focused laser beam spot $\sim 25 \mu \mathrm{m}$ and $D$ is the thermal diffusivity of a-ITO ( $\left.1.2 \times 10^{-6} \mathrm{~m}^{2} / \mathrm{s}\right)$. This value of $t_{c}$ is much larger than the time interval between successive pulses, i.e., $2 \mu$ s for a $500 \mathrm{kHz}$ repetition rate. Thus, an obvious heat accumulation effect occurred within the local region of the ITO film. In addition, in each case, it can be seen that the c-ITO line pattern

is retained on the glass substrate. This means that the irradiated laser energy is sufficient to crystallize the a-ITO thin film, and the irradiated a-ITO is completely transformed into c-ITO. In a previous study [16], it was found that the absorption coefficient $\alpha$ of an ITO thin film (thickness 120 $\mathrm{nm})$ at a wavelength of $1064 \mathrm{~nm}$ was about $5.4 \times 10^{3} \mathrm{~cm}^{-1}$, and the optical penetration depth $1 / \alpha$ was estimated to be around $1.85 \mu \mathrm{m}$. In this study, the optical penetration depth of a $1064 \mathrm{~nm}$ laser irradiation is assumed to be around 1.85 $\mu \mathrm{m}$; this leads to the conclusion that the irradiated laser energy is almost totally absorbed by the a-ITO film (thickness $\sim 100 \mathrm{~nm}$ ) itself, and that the glass substrate acts only as a heat barrier due to poor thermal conductivity. (a)

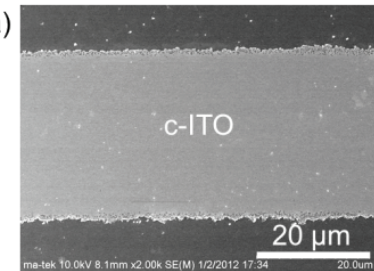

(b)

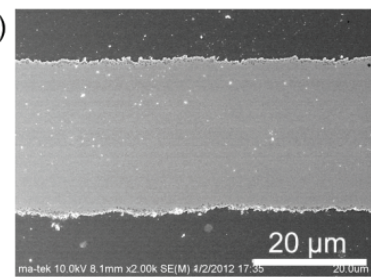

(c)

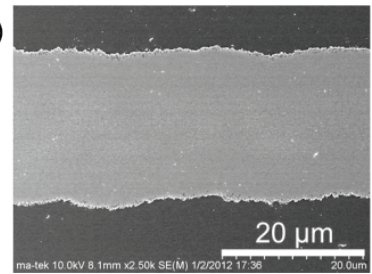

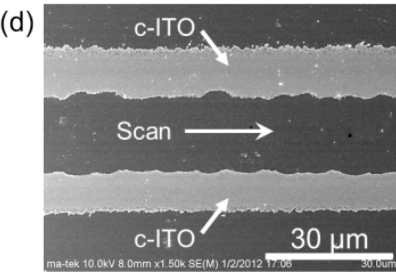

(e)

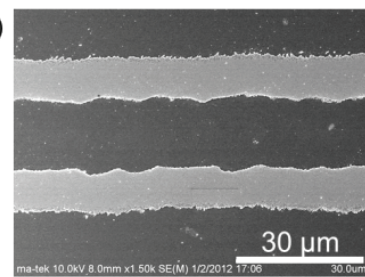

(f)

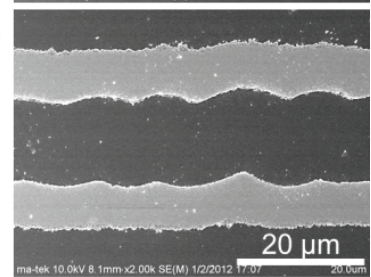

Figure 4: SEM images of Figure 3(d)-(f) and Figure 2(d)(f), respectively

In order to study the crystalline structure, optical transparency and electrical resistively of the fabricated cITO pattern, an analyzed sample was made by scanning an area $30 \times 30 \mathrm{~mm}^{2}$ with laser power of $0.33 \mathrm{~W}$, a pulse repetition rate of $500 \mathrm{kHz}$ and a scanning speed of $2.5 \mathrm{~mm} / \mathrm{s}$. Fig. 5 shows the XRD results for the as-deposited and laser-crystallized ITO films. As seen in Fig. 5(a), a-ITO thin films did not appear crystalline, and the diffusion hump at $2 \theta=24^{\circ}$ was due to the glass substrate. In Fig. 5(b), crystalline peaks of indium oxide at (222) and (440) indicated the formation of a crystalline structure after laser crystallization. Figure 6 shows the optical transmittance spectrum. As seen, the transparency in the visible range was increased from $79.2 \%$ to $82.9 \%$ at a wavelength of $550 \mathrm{~nm}$. The increase in transmittance is significant at wavelengths (350$450 \mathrm{~nm}$ ). This results primarily from the Burstein-Moss effects as the conduction band is pushed to higher energies by the carrier concentration increase [17]. The resistively of the c-ITO thin film was measured at $\sim 3.42 \times 10^{-4} \Omega-\mathrm{cm}$, which was much lower than that of the as-deposited a-ITO thin film $\sim 3.70 \times 10^{-3} \Omega-\mathrm{cm}$. 
The "ITRI 17" characters were synchronically scanned on a-ITO thin film surface using the DOE, a laser power of $0.33 \mathrm{~W}$, a pulse repetition rate of $500 \mathrm{kHz}$ and a scanning speed of $2.5 \mathrm{~mm} / \mathrm{s}$. After a chemical etching process, the microscope image of the center three "ITRI 17" characters' pattern is shown in Figure 7. It is found that three clear "ITRI 17" with c-ITO characteristics were fabricated on the glass substrate. As shown in the magnification image, because of the non-uniform energy distribution of the three divided beams after DOE and different focusing condition, the width of the line pattern is slightly varied, but the divided laser beams have sufficient laser power to crystallize the a-ITO.

(a)

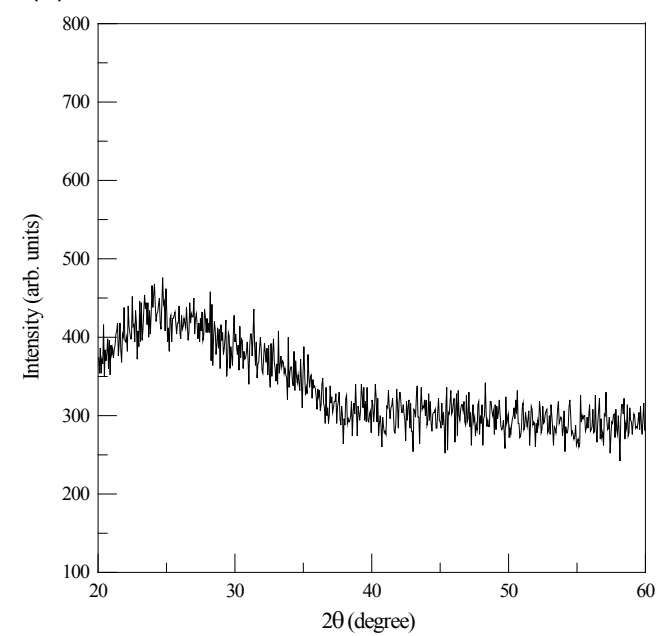

(b)

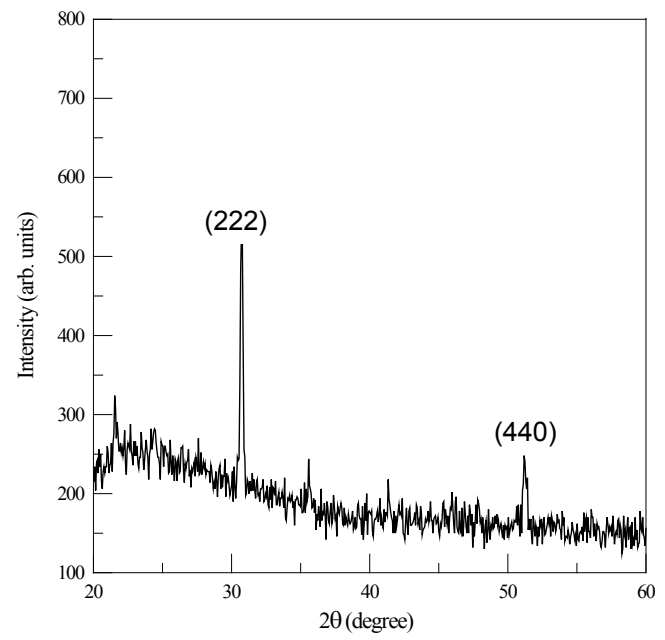

Figure 5: X-ray diffraction (XRD) result of (a) asdeposited and (b) laser-crystallized ITO films

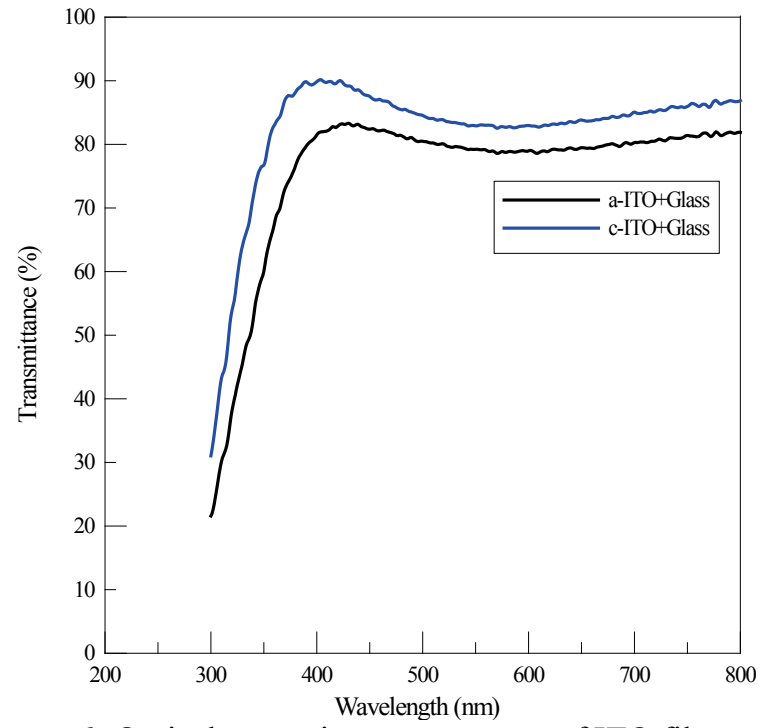

Figure 6: Optical transmittance spectrum of ITO film

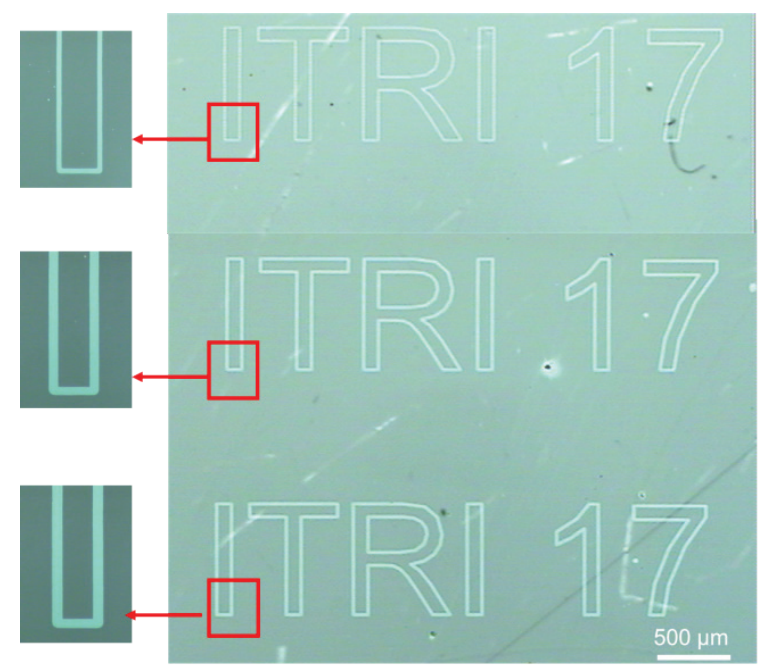

Figure 7: Microscopic image of the three "ITRI 17" characters' pattern fabricated by the DOE

\section{Conclusions}

Fabrication of micro c-ITO structures from a-ITO thin film is demonstrated using infrared picosecond fiber laserinduced crystallization followed by chemical etching. In the proposed approach, a-ITO film was irradiated over the desired area under a single laser beam (without DOE) and multiple laser beams by DOE. The irradiated substrate was then etched to remove any residual a-ITO film. By synchronizing a galvanometric scanning system, high flexibility to generate various c-ITO patterns is demonstrated.

\section{Acknowledgements}

We would like to appreciate the support from the Ministry of the Economic Affairs (MOEA), Taiwan, R.O.C. for performing this project.

\section{References}

[1] H. Morikawa, and M. Fujita, "Crystallization and electrical property change on the annealing of 
amorphous indium-oxide and indium-tin-oxide thin films," Thin Solid Films, vol. 359, no. 1, pp. 61-67, 2000.

[2] O. Yavas, and M. Takai, "High-speed maskless laser patterning of thin films for giant microelectronics," Japanese Journal of Applied Physics Part 1-Regular Papers Short Notes \& Review Papers, vol. 38, no. 12B, pp. 7131-7134, 1999.

[3] M. Y. Xu, J. Li, L. D. Lilge et al., "F-2-laser patterning of indium tin oxide (ITO) thin film on glass substrate," Applied Physics a-Materials Science \& Processing, vol. 85, no. 1, pp. 7-10, 2006.

[4] M. Park, B. H. Chon, H. S. Kim et al., "Ultrafast laser ablation of indium tin oxide thin films for organic light-emitting diode application," Optics and Lasers in Engineering, vol. 44, no. 2, pp. 138146, 2006.

[5] G. Raciukaitis, M. Brikas, M. Gedvilas et al., "Patterning of indium-tin oxide on glass with picosecond lasers," Applied Surface Science, vol. 253, no. 15, pp. 6570-6574, 2007.

[6] K. H. Kim, S. J. Kwon, H. S. Mok et al., "Laser direct patterning of indium tin oxide layer for plasma display panel bus electrode," Japanese Journal of Applied Physics Part 1-Regular Papers Brief Communications \& Review Papers, vol. 46, no. 7A, pp. 4282-4285, 2007.

[7] H. W. Choi, D. F. Farson, J. Bovatsek et al., "Direct-write patterning of indium-tin-oxide film by high pulse repetition frequency femtosecond laser ablation," Applied Optics, vol. 46, no. 23, pp. 5792-5799, 2007.

[8] Z. H. Li, E. S. Cho, and S. J. Kwon, "A new laser direct etching method of indium tin oxide electrode for application to alternative current plasma display panel," Applied Surface Science, vol. 255, no. 24, pp. 9843-9846, 2009.

[9] G. Legeay, X. Castel, R. Benzerga et al., "Excimer laser beam/ITO interaction: from laser processing to surface reaction," physica status solidi (c), vol. 5, no. 10, pp. 3248-3254, 2008.

[10] C.-W. Cheng, Y.-J. Lee, W.-C. Shen et al., "Femtosecond Laser-induced Crystallization of Amorphous Indium Tin Oxide Film on Glass Substrate for Patterning Applications," Journal of Laser Micro Nanoengineering, vol. 4, no. 3, pp. 165-169, 2009.

[11] C. W. Cheng, W. C. Shen, C. Y. Lin et al., "Fabrication of micro/nano crystalline ITO structures by femtosecond laser pulses," Applied Physics a-Materials Science \& Processing, vol. 101, no. 2, pp. 243-248, 2010.

[12] C.-W. Cheng, C.-Y. Lin, W.-C. Shen et al., "Patterning crystalline indium tin oxide by high repetition rate femtosecond laser-induced crystallization," Thin Solid Films, vol. 518, no. 23, pp. 7138-7142, 2010.

[13] H. Hosono, M. Kurita, and H. Kawazoe, "Excimer laser crystallization of amorphous indium-tinoxide and its application to fine patterning,"
Japanese Journal of Applied Physics Part 2Letters, vol. 37, no. 10A, pp. L1119-L1121, 1998.

[14] R. R. Gattass, and E. Mazur, "Femtosecond laser micromachining in transparent materials," Nature Photonics, vol. 2, no. 4, pp. 219-225, 2008.

[15] S. Juodkazis, H. Misawa, and I. Maksimov, "Thermal accumulation effect in threedimensional recording by picosecond pulses," Applied Physics Letters, vol. 85, no. 22, pp. 52395241, 2004.

[16] G. Raciukaitis, M. Brikas, M. Gedvilas et al., "Patterning of ITO Layer on Glass with High Repetition Rate Picosecond Lasers," Journal of Laser Micro Nanoengineering, vol. 2, no. 1, pp. 16, 2007.

[17] W. Chung, M. O. Thompson, P. Wickboldt et al., "Room temperature indium tin oxide by $\mathrm{XeCl}$ excimer laser annealing for flexible display," Thin Solid Films, vol. 460, no. 1-2, pp. 291-294, 2004.

(Received: June 8, 2012, Accepted: May 20, 2013) 